\title{
Using the International Classification of Functioning, Disability and Health as a tool for analysis of the effect of physical therapy on spasticity in HAM/TSP patients
}

\author{
Luana Rego Rodrigues ${ }^{[1]}$, Luzielma Macedo Glória ${ }^{[1]}$, \\ Mayara do Socorro Brito dos Santos ${ }^{[1]}$, Rita Medeiros ${ }^{[2]}$, \\ George Alberto da Silva Dias ${ }^{[1],[2]}$ and Denise da Silva Pinto ${ }^{[1]}$
}

[1]. Instituto de Ciências da Saúde, Universidade Federal do Pará, Belém, Pará, Brasil. [2]. Núcleo de Medicina Tropical, Universidade Federal do Pará, Belém, Pará, Brasil.

\begin{abstract}
Introduction: This study aimed to evaluate spasticity in human T-lymphotropic virus type 1-associated myelopathy/tropical spastic paraparesis (HAM/TSP) patients before and after physical therapy using the International Classification of Functioning, Disability and Health (ICF). Methods: Nine subjects underwent physical therapy. Spasticity was evaluated using the Modified Ashworth Scale. The obtained scores were converted into ICF body functions scores. Results: The majority of subjects had a high degree of spasticity in the quadriceps muscles. According to the ICF codes, the spasticity decreased after 20 sessions of physical therapy. Conclusions: The ICF was effective in evaluating spasticity in HAM/TSP patients.
\end{abstract}

Keywords: Tropical spastic paraparesis. ICF. Spasticity.

Human T-lymphotropic virus type 1 (HTLV-1) is a retrovirus that infects approximately 15-20 million people worldwide. HTLV-1 is endemic in several regions of the world. Thus, the virus has been detected in all Brazilian regions, with varying prevalence rates. It is estimated that approximately 2.5 million Brazilians are infected $^{(1)}$. The chronic and persistent infection of human T-cells with HTLV-1 may cause neoplastic and inflammatory diseases. Although the majority of infected individuals remain lifelong asymptomatic carriers, approximately 2-5\% develop a progressive neurological disease termed HTLV-1-associated myelopathy/ tropical spastic paraparesis $(\mathrm{HAM} / \mathrm{TSP})^{(2)}$, the most common neurological manifestation of HTLV-1. It is characterized by progressive spastic paraparesis, with the proximal muscles of the lower limbs being affected most, and neurogenic bladder disturbance ${ }^{(3)}$. HAM/TSP typically has a slow onset and chronic and steady progression.

The World Health Organization (WHO) developed the International Classification of Functioning, Disability and Health (ICF) to provide a multidimensional framework and standard language for the description of health and healthrelated components of wellbeing. The ICF has become an

Corresponding author: Dr. George Alberto da Silva Dias. Núcleo de Medicina Tropical/UFPA. Av. Generalíssimo Deodoro 92, Umarizal, 66055-240 Belém, Pará, Brasil.

Phone: 5591 98108-9582

e-mail: georgealbertodias@yahoo.com.br

Received 16 September 2014

Accepted 14 December 2014 important classification of disability and functioning, allowing an increasingly global view and positive approach to the clinical state of the individual ${ }^{(4)(5)}$. It can represent a valuable tool for assessing patients with HAM/TSP. The aim of this study was to use the ICF to evaluate spasticity in patients with HAM/TSP before and after physical therapy intervention.

This study was reviewed and approved by the Center of Tropical Medicine Committee for Ethics in Research, and all subjects signed an informed consent form (Protocol No. 063/2011). A convenience sample included nine HAM/TSP patients who were recruited from the Laboratory for Clinical and Epidemiological studies of Endemic Diseases of the Center for Tropical Medicine, Pará Federal University, Belém, Brazil (Laboratório de Clínica e Epidemiologia de Doenças Endêmicas do Núcleo de Medicina Tropical, Universidade Federal do Pará, Belém, Pará, Brazil). The patients underwent physical therapy according to the protocol described below.

The following inclusion criteria were used: 18 years of age or older; presence of HTLV-1 antibodies in serum and HTLV-1 deoxyribonucleic acid (DNA) in blood as determined with polymerase chain reaction (PCR), no co-infection with immunosuppressive viruses; and diagnosis of HAM/TSP based on the WHO criteria ${ }^{(6)}$. Individuals were excluded from the study if they had a positive PCR for HTLV-2 and if they had disorders with symptoms resembling HAM/TSP ${ }^{6}$. After initial screening of volunteers, individual interviews and physical examinations were conducted to ensure that the inclusion and exclusion criteria of the study were met. Six (66.7\%) women and three (33.3\%) men were included, and the mean age was 51.6 years. 
Spasticity was estimated using the Modified Ashworth Scale. The protocol included an initial assessment and a final assessment conducted after 20 sessions of physical therapy by the same physical therapist. Assessments were conducted with the patient in the supine position on a padded table by manually moving a lower limb through the range of motion to passively stretch specific muscle groups. The hip adductors, knee extensors, and ankle plantar flexors were tested. Passive resistance in the joint was evaluated subjectively by the examiner and given a score on a scale of resistance between 0 and 4 (Table 1).

The physical therapist was instructed to devise a treatment program with the aim of improving the subject's gait, balance, flexibility, and muscle strength. The exercises were practiced for 50 to 60 minutes once per week for 20 weeks.

Three 30 -second sessions of passive stretching were used for the hip adductors, knee flexors, and ankle plantar flexors. Passive and/or assisted hip, knee, and ankle joint mobilization was performed, using ten repetitions for each exercise.

Strength exercises were used for the upper and lower limbs and abdominal region, along with four specific proprioceptive neuromuscular facilitation (PNF) techniques: rhythmic initiation and a combination of isotonics, stabilizing reversals, and rhythmic stabilization. Two sets of five repetitions were performed.

To achieve data homogeneity and use a universal assessment scale, the Modified Ashworth Scale scores were converted into ICF body functions scores in the b7353 category (Tone of muscles of lower half of body), which the ICF outlines as follows: functions related to the tension present in the resting muscles and muscle groups in the lower half of the body and the resistance offered when trying to move those muscles passively. Inclusion: impairments associated with paraparesis and paraplegia. Qualifiers: 0, no difficulty; 1, mild difficulty; 2 , moderate difficulty; 3 , severe difficulty; 4 , complete difficulty; 8 , not specified; 9 , not applicable.

The Wilcoxon test was used to compare spasticity before and after physical therapy. Statistical analysis was performed using the BioEstat 5.0 program (available at http://www.mamiraua. org.br/downloads/programas) with the level of significance set at $5 \%$.

A descriptive analysis revealed that the great majority of subjects had a high degree of spasticity in the right quadriceps (88.8\%), followed by the left quadriceps (77.8\%) (Figure 1). When the degree of spasticity was analyzed according to the ICF codes, decreased spasticity was observed after 20 sessions of physical therapy, but this difference was only significant in the quadriceps muscles (Figure 1).

Spasticity is one of the most common disabling features of motor disorders associated with upper motor neuron syndrome ${ }^{(7)}$. It is characterized by involuntary muscle activity such as spasms, hyperreflexia, clonus, and co-contraction ${ }^{(8)}$ and is present mainly in antigravity muscles ${ }^{(9)}$. Muscle activity can be normalized through physical therapy and measured using the Modified Ashworth Scale, which is widely used for this purpose, as well as the ICF. Studies have shown that the ICF provides a scientific basis for understanding and studying health ${ }^{(10)(11)(12)}$. However, the ICF and Ashworth Scale are rarely used in HAM/ TSP patients ${ }^{(13)}$.

In agreement with the current literature ${ }^{(13)(14)}$, the vast majority of our patients showed a high degree of spasticity in the right and left quadriceps (Figure 1). We found that the ICF was useful for the evaluation of the effect of physical therapy on spasticity. Thus, the initial b7353.3 ICF code for the quadriceps muscles improved to b7353.1, reflecting a significant positive effect of the therapy (Figure 1).

Active and/or passive exercises decrease spasticity, improve balance, and preserve joint integrity. The application of our physical therapy protocol resulted in an improvement in the quality of life and reduction in morbidity from the disease. Furthermore, the abnormal sensory input and motor neuron activity ${ }^{(15)}$ were reduced more substantially when the PNF technique was utilized in this study. Therefore, strength exercises can reduce muscle spasticity and improve neural control while maintaining extensibility ${ }^{(13)}$, which was observed in the present study for the quadriceps muscles (Figure 1).

The usage of the ICF codes revealed a greater functional independence and quality of life of the patients after the therapy,

TABLE 1 - The Modified Ashworth Scale for the assessment of degree of spasticity.

\begin{tabular}{ll}
\hline Grade & Description \\
\hline 0 & No increase in muscle tone \\
1 & $\begin{array}{l}\text { Slight increase in muscle tone, manifested by a catch and release or by minimal resistance at the end of the ROM when the } \\
\text { affected part(s) is moved in flexion or extension }\end{array}$ \\
$1+$ & $\begin{array}{l}\text { Slight increase in muscle tone, manifested by a catch, followed by minimal resistance throughout the remainder (less than half) } \\
\text { of the ROM }\end{array}$ \\
3 & More marked increase in muscle tone through most of the ROM, but affected part(s) easily moved \\
4 & Considerable increase in muscle tone, passive movement difficult \\
\hline
\end{tabular}

ROM: range of motion. 


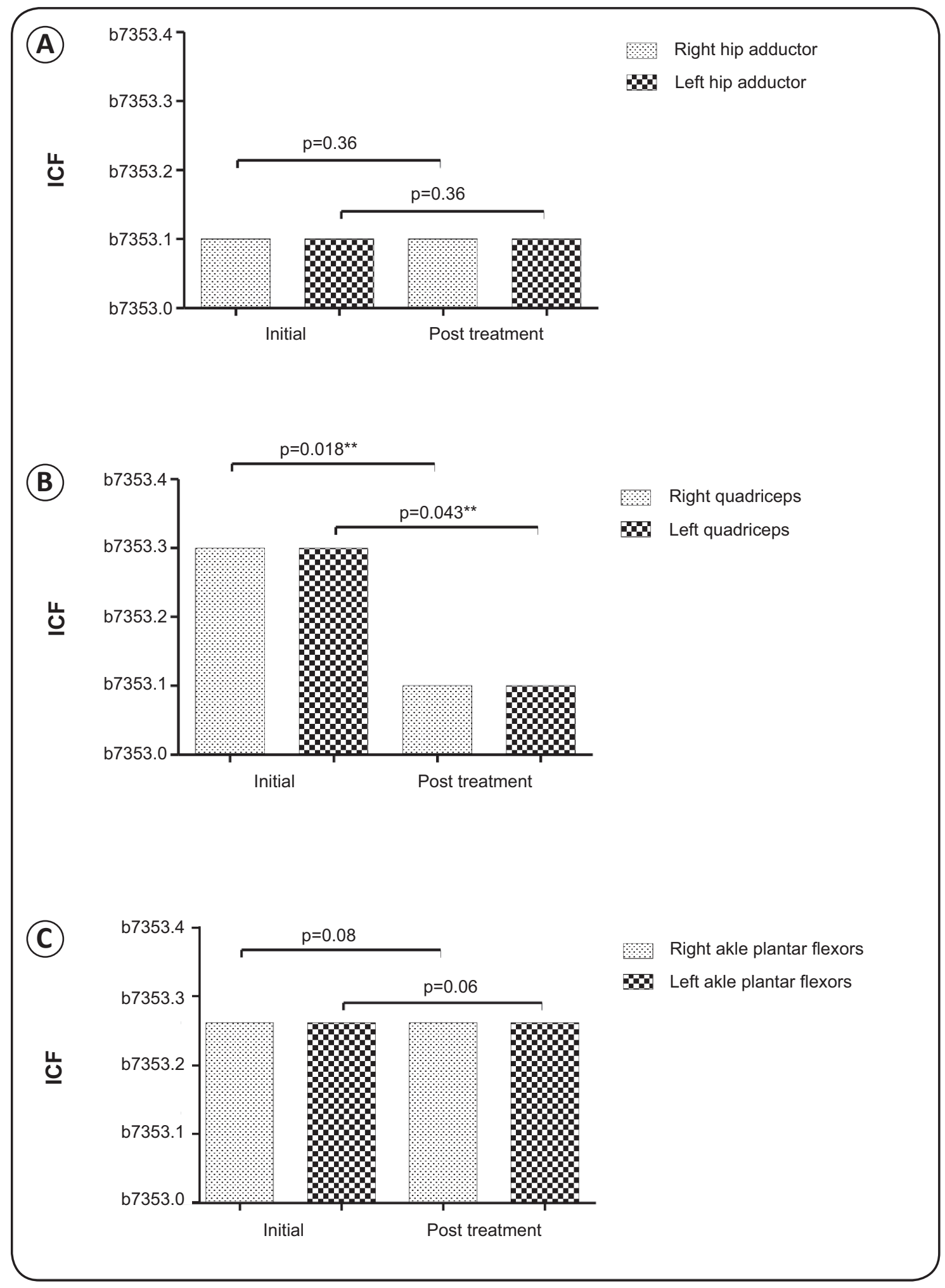

FIGURE 1 - The International Classification of Functioning, Disability and Health codes before and after the physical therapy. A: Hip adductors. B: Knee extensors. C: Ankle plantar flexors. The Wilcoxon test was used for statistical analysis. ICF: International Classification of Functioning, Disability and Health. 
emphasizing the effectiveness of physical therapy in controlling spasticity and demonstrating the value of the ICF as a tool for the evaluation of spasticity in HAM/TSP patients. This confirms that the ICF is a universal and standardized language that provides a scientific basis for communication between health professionals and researchers.

\section{CONFLICT OF INTEREST}

The authors declare that there is no conflict of interest.

\section{REFERENCES}

1. Dourado L, Alcantara LC, Barreto ML, da Gloria Teixeira M, Galvão-Castro B. HTLV-I in the general population of Salvador, Brazil: a city with African ethnic and sociodemographic characteristics. J Acquir Immune Defic Syndr 2003; 34:527-531.

2. Oliére S, Douville R, Sze A, Belgnaoui SM, Hiscott J. Modulation of innate immune responses during human T-cell leukemia virus (HTLV-1) pathogenesis. Cytokine Growth Factor Rev 2011; 22:197-210

3. Shoeibi A, Etemadi M, Moghaddam Ahmadi A, Amini M, Boostani R. "HTLV-I Infection" twenty-year research in neurology department of mashhad university of medical sciences. Iran J Basic Med Sci 2013; 16:202-207.

4. Fairbairn K, May K, Yang Y, Balasundar S, Hefford C, Abbott JH. Mapping patient-specific functional scale (PSFS) items to the international classification of functioning, disability and health (ICF). Phys Ther 2012; 92:310-317.

5. Stucki G, Kostanjsek N, Üstün B, Cieza A. ICF-based classification and measurement of functioning. Eur J Phys Rehabil Med 2008; 44:315-328.
6. De Castro-Costa CM, Araújo AQ, Barreto MM, Takayanagui OM, Sohler MP, da Silva EL, et al. Proposal for diagnostic criteria of tropical spastic paraparesis/HTLV-I-associated myelopathy (TSP/HAM). AIDS Res Hum Retroviruses 2006; 22:931-935.

7. Pandyan AD, Gregoric M, Barnes MP, Wood D, Van Wijck F, Burridge J, et al. Spasticity: clinical perceptions, neurological realities and meaningful measurement. Disabil Rehabil 2005; 27:2-6.

8. D'Amico JM, Condliffe EG, Martins KJ, Bennett DJ, Gorassini MA. Recovery of neuronal and network excitability after spinal cord injury and implications for spasticity. Front Integr Neurosci 2014; 12:36.

9. Dietz V, Sinkjaer T. Spastic movement disorder: impaired reflex function and altered muscle mechanics. Lancet Neurol 2007; 6:725-733.

10. Cieza A, Stucki G. The international classification of functioning disability and health: its development process and content validity. Eur J Phys Rehabil Med 2008; 44:303-313.

11. Fréz AR, Vignola BAP, Kaziyama HHS, Spezzano LC, Filippo TRM, Imamura M, et al. The relationship between the functional independence measure and the international classification of functioning, disability, and health core set for stroke. Acta Fisiatr 2013; 20:24-28.

12. Stucki A, Cieza A, Michel F, Stucki G, Bentley A, Culebras A, et al. Developing ICF core sets for persons with sleep disorders based on the international classification of functioning, disability and health. Sleep Med 2008; 9:191-198.

13. Britto VL, Correa R, Vincent MB. Proprioceptive neuromuscular facilitation in HTLV-I-associated myelopathy/tropical spastic paraparesis. Rev Soc Bras Med Trop 2014; 47:24-29.

14. Franzoi AC, Araújo AQC. Disability profile of patients with HTLV-I associated myelopathy/tropical spastic paraparesis using the functional independence measure (FIM). Spinal Cord 2005; 43:236-240.

15. Gracies JM. Pathophysiology of impairment in patients with spasticity and use of stretch as a treatment of spastic hypertonia. Phys Med Rehabil Clin N Am 2001; 12:747-768. 